\title{
树鼠解剖数据的测定与分析
}

\author{
李 波 ${ }^{1,2}$, 张荣平 ${ }^{1}$, 李进涛 ${ }^{1,2}$, 何保丽 ${ }^{1,2}$, 郑 红 $^{1,2}$, 王利梅 ${ }^{1}$, 角建林 ${ }^{1, *}$ \\ 1. 昆明医科大学 实验动物学部, 云南 昆明 650031; \\ 2. 扬州大学 兽医学院, 江苏 扬州 225009
}

\begin{abstract}
摘要: 解剖数据是实验动物主要的生物学特性指标。该文对实验室驯养树鼠 (7 9 月龄)的体尺、骨骼、乳头、肠道及脏器 重量与系数等解剖学数据进行了测定与分析。31 项解剖数据测量结果显示雌、雄个体间体高、右耳宽、回肠及结肠差异显 著 $(P<0.05)$, 体斜长、胸深、躯干长、左右两前肢长、右后肢长、左右两侧耳长、左耳宽、龙骨长、左右两侧胫长、十二 指肠及空肠长等差异极显著 $(P<0.01)$ 。以体长为因变量, 尾长、躯干长、左前肢长、右前肢长、左后肢长及右后肢长等为 自变量作逐步回归分析, 回归方程为: 体长 $=13.90+$ 尾长 $\times 0.16$ 。37 项脏器及系数测定结果: 雌雄间比较, 体重、心、肺、脾、 左肾、右肾、膀胱、左海马、右海马、左领下腺、左甲状腺、右甲状腺重量差异极显著 $(P<0.01)$ 。 小肠、右领下腺、左肾上 腺之间差异显著 $(\mathrm{P}<0.05)$; 心、肺、胃、膀胱、小肠、大肠、脑、右海马、左肾上腺系数雌雄间差异极显著 $(P<0.01)$ 。右肾、 左海马、左领下腺、右肾上腺、左右两侧甲状腺系数之间达到了显著性水平 $(P<0.05)$ 。以动物体重为因变量, 以主要脏器 指标: 心脏、肺、肝、脾、左肾、右肾、脑为自变量, 作逐步回归分析, 回归方程为: 体重 $=62.73+$ 左肾 $\times 79.213+$ 心脏 $\times 24.09$ 。 实验室驯养树鼠不同性别对体尺、脏器及系数、肠道等解剖数据有一定影响, 为树鼠实验动物化及人类疾病动物模型研究提 供基础数据。
\end{abstract}

关键词: 树鼠; 解剖数据; 脏器重量; 脏器系数

中图分类号: Q4 文献标志码: A 文章编号: 0254-5853-(2013)02-0132-07

\section{Measurement and analysis of anatomical parameter values in tree shrews}

\author{
Bo LI ${ }^{1,2}$, Rong-Ping ZHANG ${ }^{1}$, Jin-Tao LI ${ }^{1,2}$, Bao-Li HE ${ }^{1,2}$, Hong ZHEN ${ }^{1,2}$, Li-Mei WANG ${ }^{1}$, \\ Jian-Lin JIAO ${ }^{1, *}$ \\ 1. Laboratory Animal Center of Kunming Medical University, Kunming 650031, China; \\ 2. College of Veterinary Medicine, Yangzhou University, Yangzhou 225009, China
}

\begin{abstract}
Anatomical parameter values in tree shrews are major biological characteristic indicators in laboratory animals. Body size, bones and mammilla, organ weights, coefficient intestinal canal and other anatomical data were measured and analyzed in laboratory domesticated tree shrews ( 7 to 9 months of age). Measurement of 31 anatomical parameters showed that body height, width of the right ear, ileum and colon had significant differences between males and females $(P<0.05)$. Highly significant differences were also found in body slanting length, chest depth, torso length, left and right forelimb length, right hind limb length, left and right ear length, left ear width, keel bone length, left and right tibia length, duodenum and jejunum $(P<0.01)$. With body length as the dependent variable, and tail length, torso length, right and left forelimb length, and left and right hind limb length as independent variables for stepwise regression analysis, the regression equation for body length $=13.90+$ tail length $\times 0.16$. The results of 37 organs weights between female and male tree shrews showed very significant differences $(P<0.01)$ for weight of heart, lungs, spleen, left and right kidney, bladder, left and right hippocampus, left submandibular gland, and left and right thyroid gland, as well as significant $(P<0.05)$ differences in the small intestine, right submandibular gland, and left adrenal gland. The coefficient of heart, lung, stomach, bladder, small and large intestine, brain, right hippocampus, and left adrenal gland showed highly significant differences $(P<0.01)$, while differences in the right kidney, left hippocampus, left submandibular gland, right adrenal gland, and left and right thyroid gland were significant $(P<0.05)$. With animal weight as the dependent variable and indicators of heart, lung, liver, spleen, left and right kidney and brain as independent variables for stepwise regression analysis, the regression equation showed that weight $=62.73+$ left kidney
\end{abstract}

收稿日期: 2012-12-12; 接受日期：2013-01-30

基金项目：云南省教育厅科学研究基金 (2012C015); 云南省科技基础条件平台建设计划 (2006PT07-1); 国家科技支撑计划 (2009BAI83B02)

*通信作者(Corresponding author), E-mail: jiaojianlin66@163.com

第一作者简介: 李波 (1979-), 实验师, 在读博士, E-mail:libo0302@sina.com. 
$\times 79.21+$ heart $\times 24.09$. Female and male laboratory domesticated tree shrews showed certain influences in body size, organ weight and coefficient, and intestinal canal regarding anatomical parameters. This experiment provides basic data for studies on laboratory tree shrews and animal models.

Keywords: Tree shrew; Anatomical parameter; Weight of organ; Organ coefficient

解剖数据作为实验动物主要的生物学特性, 是 直接描述动物体征的主要数据群。灵长类近亲树鼠 (tree shrew, Tupaia belangeri) 为单系起源的攀鼠目 (Peng et al, 1991; Nie et al, 2008; Dong et al, 2012; Xu et al, 2013), 由于其特殊的生物学特性, 已被广 泛应用于医学和生物学研究, WHO 也曾建议用树 鼠代替濒危的非人灵长类动物进行科学实验 (Peng et al, 1991)。近年来, 树鼠实验室规模化驯养繁殖 的成功 (Jiang et al, 2011; Jiao et al, 2009; Shen et al, 2011)加快了其实验动物化的进程。本研究对实验室 驯养树鼠的解剖学数据进行了全面测定与分析, 为 树鼠实验动物化提供基础数据。

\section{1 材料和方法}

\section{1 动物来源}

实验室驯养 7 9 月龄树舄 70 只, 雌、雄各半, 由昆明医科大学实验动物学部提供。实验动物使用 符合国家和学校动物伦理委员会规定并遵守国际 惯例。

\section{2 仪器、试剂及测定方法}

解剖前, 动物禁食不禁水 $12 \mathrm{~h}$, 称重 (体重计 购自上海精密科学仪器有限公司, YP2001N)后, 腹 腔注射戊巴比妥钠盐 $35 \mathrm{mg} / \mathrm{kg}$ (北京鼎国昌盛生物 技术有限责任公司, 批号: 20120812), 麻醉后心脏 采血、处死。实验参照《啮齿类实验动物生物学特 性数据测定技术规程》(He et al, 2006) 相关要求进 行。获取体尺(软尺测量)及骨骼等解剖数据后依次 采集腹腔、胸腔及脑等器官。采集脏器时需将周围 脂肪及结缔组织除尽, 滤纸吸干表面的血液和体 液, 腔性器官需在去除腔内液体和内容物后迅速称 重 (梅特勒一托利多 AL204-IC 电子天平)。脏器系 数 $=$ 脏器重量 / 体重 $\times 100 \%$ 。剖取从胃幽门处至肛 门处的所有肠段, 置于盛有生理盐水的平血中并在 硫酸纸上展开, 分别测量十二指肠、空肠、回肠、 盲肠、结肠及直肠等的长度。多个肠道需同时测量 并由一人操作整个过程, 避免由人为过度展开或展 开不充分而造成测量数据不准确。

\section{3 统计学方法}

采用 SPSS11.5 软件包进行分析。正态计量资料 采用 $t$-test，非正态用秩和检验。

\section{2 结 果}

\section{1 解剖数据}

树鼣有 7 个颈椎、 13 个胸椎、 6 个腰椎、 3 个 骮椎、24 27 个尾椎及 6 个乳头。雌、雄个体体长、 尾长、尾/体比、胸宽、左后肢长以及盲肠和直肠长 无显著性差异 $(P>0.05)$; 体高、右耳宽、回肠及结 肠长差异显著 $(P<0.05)$; 体斜长、胸深、躯干长、 左前肢长、右前肢长、右后肢长、左耳长、右耳长、 左耳宽、龙骨长、左胫长、右胫长、十二指肠及空 肠长等差异极显著 $(P<0.01)$ (表 1, 图 1)。

\section{2 体尺指标的相关与回归分析}

部分树鼠体尺数据的相关分析见表 2。以体长 为因变量, 部分体尺指标 (尾长、躯干长、左前肢 长、右前肢长、左后肢长及右后肢长等)为自变量, 作逐步回归分析, 回归方程为: 体长 $=13.90+$ 尾长 $\times 0.16$ 。

\section{3 脏器及其系数测定}

7 9 月龄树舄䧳、雄个体体重差异极显著 $(P<$ $0.01)$; 心、肺、膀胱及右海马重量及系数差异极显 著 $(P<0.01)$; 右肾、左海马、左领下腺、左右甲状 腺重量差异极显著 $(P<0.01)$, 系数差异显著 $(P<0.05)$; 小肠及左肾上腺重量差异显著 $(P<0.05)$, 系数差异极显著 $(P<0.01)$; 脾及左肾重量差异极显 著 $(P<0.01)$, 但系数无差异; 右领下腺重量差异显 著 $(P<0.05)$, 但系数无差异; 胃、大肠及脑重量无 差异, 系数差异极显著 $(P<0.01)$; 右肾上腺重量无 差异, 系数差异显著 $(P<0.05)$ 。

\section{4 主要脏器指标的相关与回归分析}

树鼠主要脏器测定数据的相关分析见表 4。以 体重为因变量, 主要脏器指标(心、肺、肝、脾、左 右肾及脑等)为自变量, 作逐步回归分析, 回归方程 为: 体重 $=62.73+$ 左肾 $\times 79.21+$ 心脏 $\times 24.09$ 。 
表 1 树鼠雌、雄个体解剖数据测量结果

Table 1 Measurement of anatomical parameters in tree shrew

\begin{tabular}{|c|c|c|c|c|c|c|c|c|c|c|c|}
\hline \multirow{3}{*}{$\begin{array}{c}\text { 测量项目 }(\mathrm{cm}) \\
\text { Item }(\mathrm{cm})\end{array}$} & \multicolumn{5}{|c|}{ 雌 Female (ㅇ) $n=35$} & \multicolumn{5}{|c|}{ 雄 Male $\left(\delta^{\Uparrow}\right) n=35$} & \multirow{3}{*}{$P$} \\
\hline & \multirow{2}{*}{$\frac{\text { 平均值 }}{\text { Mean }}$} & \multirow{2}{*}{$\frac{\text { 标准差 }}{S D}$} & \multirow{2}{*}{$\frac{\text { 中位数 }}{\text { Medium }}$} & \multicolumn{2}{|c|}{ 四分位数 } & \multirow{2}{*}{$\frac{\text { 平均值 }}{\text { Mean }}$} & \multirow{2}{*}{$\frac{\text { 标准差 }}{S D}$} & \multirow{2}{*}{$\frac{\text { 中位数 }}{\text { Medium }}$} & \multicolumn{2}{|c|}{ 四分位数 } & \\
\hline & & & & $\mathrm{D}_{\mathrm{L}}$ & $\mathrm{D}_{\mathrm{U}}$ & & & & $\mathrm{D}_{\mathrm{L}}$ & $\mathrm{D}_{\mathrm{U}}$ & \\
\hline 体高 Body height & 7.20 & 0.55 & 7.20 & 7.00 & 7.50 & 6.93 & 0.25 & 7.00 & 6.80 & 7.10 & $*$ \\
\hline 体长 Body length & 16.68 & 0.48 & 16.50 & 16.50 & 17.00 & 16.59 & 0.43 & 16.60 & 16.40 & 16.80 & \\
\hline 尾长 Tail length & 16.73 & 0.71 & 17.00 & 16.50 & 17.00 & 16.85 & 0.64 & 17.00 & 16.50 & 17.30 & \\
\hline 尾/体比 Tail/Body & 1.00 & 0.05 & 1.01 & 0.97 & 1.03 & 1.02 & 0.04 & 1.02 & 0.98 & 1.04 & \\
\hline 体斜长 Body slanting length & 10.67 & 0.38 & 10.60 & 10.50 & 11.00 & 11.38 & 0.39 & 11.50 & 11.10 & 11.70 & $* *$ \\
\hline 胸宽 Chest width & - & - & 3.20 & 3.10 & 3.40 & - & - & 3.40 & 3.20 & 3.50 & \\
\hline 胸深 Chest depth & 4.88 & 0.37 & 5.00 & 4.70 & 5.00 & - & - & 5.40 & 5.20 & 5.50 & $* *$ \\
\hline 躯干长 Torso length & - & - & 9.00 & 9.00 & 10.00 & 10.18 & 0.55 & 10.30 & 9.90 & 10.50 & $* *$ \\
\hline 左前肢长 Left forelimb length & - & - & 9.00 & 8.70 & 9.20 & 9.47 & 0.33 & 9.40 & 9.20 & 9.80 & $* *$ \\
\hline 右前肢长 Right forelimb length & - & - & 9.00 & 8.80 & 9.20 & 9.31 & 0.36 & 9.30 & 9.10 & 9.50 & $* *$ \\
\hline 左后肢长 Left hind limb length & 11.07 & 0.32 & 11.00 & 11.00 & 11.30 & 11.20 & 0.51 & 11.30 & 11.10 & 11.40 & \\
\hline 右后肢长 Right hind limb length & 11.08 & 0.30 & 11.00 & 11.00 & 11.20 & 11.30 & 0.25 & 11.30 & 11.10 & 11.50 & $* *$ \\
\hline 左耳长 Left ear length & 1.56 & 0.23 & 1.60 & 1.50 & 1.70 & - & - & 1.20 & 1.10 & 1.20 & $* *$ \\
\hline 右耳长 Right ear length & 1.56 & 0.20 & 1.60 & 1.50 & 1.70 & - & - & 1.20 & 1.10 & 1.20 & $* *$ \\
\hline 左耳宽 Left ear width & 1.55 & 0.14 & 1.50 & 1.50 & 1.70 & - & - & 1.70 & 1.60 & 1.70 & $* *$ \\
\hline 右耳宽 Right ear width & 1.58 & 0.14 & 1.60 & 1.50 & 1.70 & - & - & 1.70 & 1.60 & 1.70 & * \\
\hline 龙骨长 Keel bone length & 1.04 & 0.13 & 1.10 & 0.90 & 1.10 & - & - & 1.20 & 1.10 & 1.20 & $* *$ \\
\hline 左胫长 Left tibia length & 3.55 & 0.23 & 3.50 & 3.40 & 3.70 & - & - & 3.20 & 3.10 & 3.30 & $* *$ \\
\hline 右胫长 Right tibia length & 3.54 & 0.26 & 3.50 & 3.30 & 3.80 & - & - & 3.20 & 3.10 & 3.30 & $* *$ \\
\hline 十二指肠长 Duodenum length & 10.66 & 2.37 & 11.00 & 9.50 & 12.00 & 12.33 & 2.67 & 12.00 & 10.00 & 14.00 & $* *$ \\
\hline 空肠长 Jejunum length & 47.76 & 5.13 & 48.00 & 44.00 & 50.00 & 39.70 & 7.08 & 40.00 & 32.00 & 46.00 & $* *$ \\
\hline 回肠长 Ileum length & 25.68 & 5.00 & 27.00 & 22.00 & 28.00 & 23.11 & 4.07 & 23.00 & 20.40 & 25.00 & $*$ \\
\hline 盲肠长 Cecum length & 2.19 & 0.36 & 2.20 & 2.00 & 2.50 & 2.24 & 0.37 & 2.20 & 2.00 & 2.50 & \\
\hline 结肠长 Colon length & 5.99 & 1.14 & 6.00 & 5.00 & 7.00 & 5.42 & 0.78 & 5.20 & 5.00 & 6.00 & * \\
\hline 直肠长 Rectum length & 1.75 & 0.48 & 1.70 & 1.50 & 2.10 & 1.69 & 0.41 & 1.60 & 1.50 & 2.00 & \\
\hline 左子宫体长 Left uterine body length & - & - & 1.00 & 0.90 & 1.10 & - & - & - & - & - & - \\
\hline 左子宫角长 Left uterine horns length & 1.32 & 0.24 & 1.25 & 1.10 & 1.50 & - & - & - & - & - & - \\
\hline 右子宫体长 Right uterine body length & 1.07 & 0.26 & 1.00 & 0.90 & 1.10 & - & - & - & - & - & - \\
\hline 右子宫角长 Right uterine horns length & 1.29 & 0.26 & 1.20 & 1.10 & 1.50 & - & - & - & - & - & - \\
\hline 阴道长 Vagina length & 2.10 & 0.58 & 2.00 & 1.70 & 2.45 & - & - & - & - & - & - \\
\hline 阴茎长 Penis length & - & - & - & - & - & 3.57 & 0.33 & 3.50 & 3.30 & 3.80 & \\
\hline
\end{tabular}

“一”: 由于无对应值或数据非正态分布产生的统计缺省值。

“_": missing values because of no corresponding or data non-normal distribution. *: $P<0.05 ; * *: P<0.01$.

\section{3 讨 论}

解剖数据是药物安全性评价实验及病原微生 物感染的检测指标，可以客观反映实验动物生长发 育程度、病变程度和功能状态等 (Sun et al, 2009), 对其种属标准化鉴定亦有一定意义 ( Ye et al,
2011)。本研究丰富了中缅树鼠的解剖数据, 为其实 验动物化提供基础数据。结果显示 7 9 月龄树鼠的 绝大部分体尺、脏器及系数指标因性别不同而存在 显著或极显著性差异, 与 Ye et al (2011)报道的野生 成年树鼠结果基本一致。因此, 若无特殊情况, 在 实验中应选择雌、雄个体各半, 并分别对其 


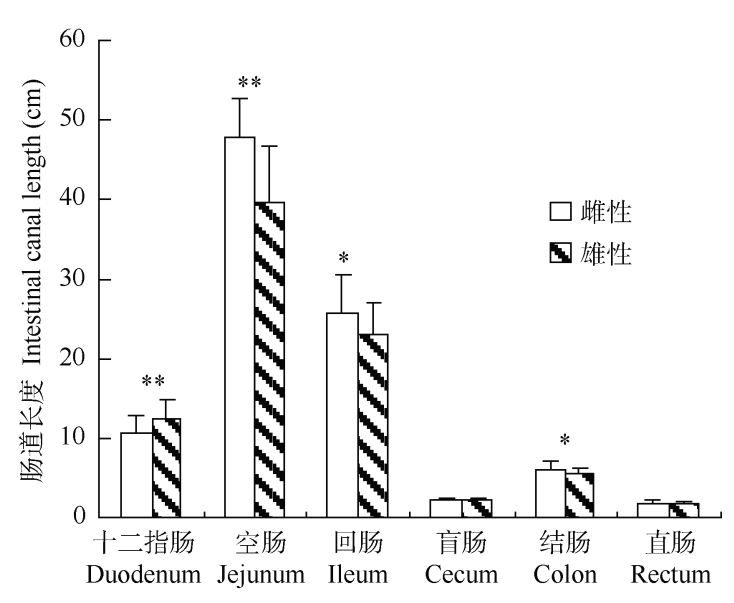

图 1 树舄雌、雄个体肠道长度比较

Figure 1 Comparison of intestinal canal length (comparison between males and females, $*: P<0.05 ; * *: P<0.01)$

进行统计分析和比较, 研究结果与 Peng et al(1991) 报道的解剖数据比较, 绝大部分相近 (如体长、尾 长、耳长、脾脏重、肾脏重、脑重、肾上腺重、卵 巢重及睪丸脏器系数等)。差异较大的是脑垂体重 量, 由于脑垂体体积较小, 故该差异可能是由称量 仪器与取材不同所导致。

小型哺乳动物的消化道长度和重量与其食性 和食物质量密切相关 (Gross et al, 1985; Korn, 1992; Derting \& Bogue, 1993)。小肠是食物消化和营养吸 收的主要部位, 在能量消耗增加或食物质量降低 时, 树鼠小肠长度及重量的增加可获得更多的能量 以满足生存需要 (Cai et al, 2010)。本研究结果显示 雌、雄个体的小肠各肠段均存在差异 $(P<0.05)$, 雌 性小肠重量显著高于雄性 $(P<0.05)$ 。因此, 在实验 室条件下, 雌、雄个体对食物的消化和吸收能力是 否存在差异仍有待进一步研究。

本研究结果显示雌、雄个体脑重量差异不显著
$(P>0.05)$, 系数差异显著 $(P<0.05)$, 体重差异极显 著 $(P<0.01)$, 与 Ye et al (2011) 的结果相反, 这可能 与个体发育阶段及生活环境相关。野生树獡的确切 年龄较难掌握, 且其机体受季节及营养状况的影响 较大 (Cao, 1989; Jiao et al, 2011; Li et al, 1994; Li et $\mathrm{al}, 2011$ )。实验动物随年龄的增长, 其脑、心、 肺、脾及肾等主要脏器系数都呈下降趋势 (Sun et al, 2009)。提示若能开展多年龄段或采用动态观测方法 检测树鼠脏器重量及生长发育情况, 将更有助于建 立其基础生物学数据。

在脏器及其系数的测定分析中, 与国内近年较 少的研究结果相比 (He et al, 2008; Ye et al, 2011), 个体心脏、肝脏、肺脏、胰腺、脑及肠等均存在差 异。其中, 雌、雄个体心脏重量与系数差异极显著 $(P<0.01)$, 这与野生成年个体情况一致 (Ye et al, 2011), 但重量较轻, 而肝脏重量小, 肤腺重量大, 且其重量与系数差异均不显著 $(P>0.05)$ 。以上结果 的差异可能由多方面原因导致: (1)研究对象、饲养 环境及饲养条件等客观条件不同，本研究采用 7 9 月龄树鼠, 与前期工作选用个体的年龄存在差异, 且所选树鼠主要在实验室条件下以颗粒饲料喂养, 与前期驯养及野外条件存在根本的不同; (2)个体的 处死及测定方法不同, 动物致死时, 心脏及肝脏等 器官内血液的充盈程度将直接影响其重量, 因此, 采集内脏器官应在采血后, 且不同的采血部位也会 影响脏器重量, 本研究麻醉后心脏采血. 5 8 $\mathrm{mL}$ 的 致死方法可能也是导致差异的原因之一; (3)结缔组 织及血管等分离情况不同; (4)研究对象不同的生长 发育阶段导致能量代谢及积蓄不同; (5)样本量不 同, Ye et al (2011)使用雄性 25 只, 雌性 35 只, He et al (2008)使用雄性 12 只、雌性 15 只, 而本研究 使用雄、雌性各 35 只。

表 2 部分树鼠体尺指标的 Pearson 相关分析

Table 2 Pearson correlation of body size in tree shrew

\begin{tabular}{|c|c|c|c|c|c|c|c|}
\hline $\begin{array}{c}\text { 项目 } / P \text { 值 } \\
\text { Item } / P \text { level }\end{array}$ & $\begin{array}{c}\text { 体长 } \\
\text { Body length }\end{array}$ & $\begin{array}{c}\text { 尾长 } \\
\text { Tail length }\end{array}$ & $\begin{array}{c}\text { 躯干长 } \\
\text { Torso length }\end{array}$ & $\begin{array}{c}\text { 左前肢长 } \\
\text { Left forelimb length }\end{array}$ & $\begin{array}{l}\text { 右前肢长 Right } \\
\text { forelimb length }\end{array}$ & $\begin{array}{c}\text { 左后肢长 Left } \\
\text { hind limb length }\end{array}$ & $\begin{array}{l}\text { 右后肢长 Right } \\
\text { hind limb length }\end{array}$ \\
\hline 体长 Body length & 1 & $0.242 *$ & 0.194 & 0.159 & 0.153 & 0.217 & -0.002 \\
\hline 尾长 Tail length & $0.242 *$ & 1 & -0.065 & $0.355^{* *}$ & $0.344^{* *}$ & 0.226 & 0.158 \\
\hline 躯干长 Torso length & 0.194 & -0.065 & 1 & 0.201 & 0.181 & $0.247 *$ & $0.331^{* *}$ \\
\hline 左前肢长 Left forelimb length & 0.159 & $0.355^{* *}$ & 0.201 & 1 & $0.917 * *$ & 0.173 & $0.269^{*}$ \\
\hline 右前肢长 Right forelimb length & 0.153 & $0.344 * *$ & 0.181 & $0.917 * *$ & 1 & 0.123 & 0.225 \\
\hline 左后肢长 Left hind limb length & 0.217 & 0.226 & $0.247 *$ & 0.173 & 0.123 & 1 & $0.480^{* *}$ \\
\hline 右后肢长 Right hind limb length & -0.002 & 0.158 & $0.331 * *$ & $0.269 *$ & 0.225 & $0.480^{* *}$ & 1 \\
\hline
\end{tabular}

$n=70 ; *: P<0.05 ; * *: P<0.01$ 
表 3 树鼠雌、雄个体部分脏器测定结果 $($ mean $\pm S D)$

Table 3 Measurement of body and organ weight and coefficient in tree shrews

\begin{tabular}{|c|c|c|c|c|c|c|}
\hline \multirow{2}{*}{$\begin{array}{l}\text { 测定项目 } \\
\text { Item }\end{array}$} & \multicolumn{2}{|c|}{ 雌 Female(只) $n=35$} & \multicolumn{2}{|c|}{ 雄 Male $\left(ठ^{\circledR}\right) n=35$} & \multirow[b]{2}{*}{$P_{1}$} & \multirow[b]{2}{*}{$P_{2}$} \\
\hline & $\begin{array}{c}\text { 脏器重量 (g) } \\
\text { Organ weight (g) }\end{array}$ & $\begin{array}{c}\text { 脏器系数 }(\%) \\
\text { Organ coefficient }(\%)\end{array}$ & $\begin{array}{c}\text { 脏器重量 (g) } \\
\text { Organ weight (g) }\end{array}$ & $\begin{array}{c}\text { 脏器系数 }(\%) \\
\text { Organ coefficient }(\%)\end{array}$ & & \\
\hline 体重 Body weight & $115.51 \pm 5.43$ & - & $125.82 \pm 11.28$ & - & $* *$ & \\
\hline 心脏 Heart & $0.62 \pm 0.09$ & $0.54 \pm 0.07$ & $0.85 \pm 0.12$ & $0.68 \pm 0.08$ & $* *$ & $* *$ \\
\hline 肺脏 Lung & $1.06 \pm 0.17$ & $0.92 \pm 0.15$ & $1.27 \pm 0.16$ & $1.02 \pm 0.13$ & $* *$ & $* *$ \\
\hline 肝脏 Liver & $3.86 \pm 0.53$ & $3.35 \pm 0.52$ & $4.09 \pm 0.51$ & $3.25 \pm 0.31$ & & \\
\hline 脾脏 Spleen & $(0.13)$ & $(0.11)$ & $0.15 \pm 0.03$ & $0.12 \pm 0.02$ & $* *$ & \\
\hline 左肾 Left kidney & $0.48 \pm 0.05$ & $0.41 \pm 0.04$ & $0.54 \pm 0.06$ & $0.43 \pm 0.04$ & $* *$ & \\
\hline 右肾 Right kidney & $0.47 \pm 0.05$ & $0.40 \pm 0.04$ & $0.53 \pm 0.07$ & $0.42 \pm 0.04$ & $* *$ & $*$ \\
\hline 胃 Stomach & $0.91 \pm 0.09$ & $0.79 \pm 0.09$ & $0.90 \pm 0.10$ & $0.72 \pm 0.10$ & & $* *$ \\
\hline 膀胱 Bladder & $0.08 \pm 0.02$ & $(0.07)$ & $0.13 \pm 0.03$ & $0.10 \pm 0.02$ & $* *$ & $* *$ \\
\hline 小肠 Small intestine & $4.20 \pm 0.73$ & $3.65 \pm 0.66$ & $3.83 \pm 0.74$ & $3.08 \pm 0.66$ & $*$ & ** \\
\hline 大肠 Large intestine & $2.02 \pm 0.45$ & $1.75 \pm 0.06$ & $1.86 \pm 0.4$ & $1.49 \pm 0.33$ & & ** \\
\hline 脑 Brain & $2.97 \pm 0.18$ & $2.58 \pm 0.19$ & $3.05 \pm 0.19$ & $2.44 \pm 0.21$ & & $* *$ \\
\hline 小脑 Cerebellum & $0.50 \pm 0.07$ & $0.44 \pm 0.07$ & $0.52 \pm 0.09$ & $0.41 \pm 0.08$ & & \\
\hline 左海马 Hippocampus & $0.09 \pm 0.04$ & $0.08 \pm 0.03$ & $0.12 \pm 0.02$ & $0.09 \pm 0.02$ & $* *$ & $*$ \\
\hline 右海马 Hippocampus & $0.08 \pm 0.02$ & $0.07 \pm 0.02$ & $0.12 \pm 0.03$ & $0.09 \pm 0.03$ & $* *$ & ** \\
\hline 脑垂体 Pituitary & $(0.0038)$ & $(0.0033)$ & $0.0046 \pm 0.0027$ & $0.0037 \pm 0.0023$ & & \\
\hline 左领下腺 Left submaxillary gland & $0.18 \pm 0.03$ & $0.15 \pm 0.03$ & $(0.21)$ & $(0.17)$ & $* *$ & $*$ \\
\hline 右领下腺 Right submaxillary gland & $0.17 \pm 0.03$ & $0.15 \pm 0.02$ & $0.20 \pm 0.05$ & $0.16 \pm 0.04$ & $*$ & \\
\hline 左腮腺 Left parotid & $0.16 \pm 0.03$ & $0.14 \pm 0.03$ & $0.17 \pm 0.04$ & $0.13 \pm 0.04$ & & \\
\hline 右腮腺 Right parotid & $0.16 \pm 0.03$ & $0.14 \pm 0.03$ & $0.16 \pm 0.04$ & $0.13 \pm 0.04$ & & \\
\hline 胸腺 Thymus & $0.05 \pm 0.03$ & $0.05 \pm 0.02$ & $0.06 \pm 0.02$ & $0.05 \pm 0.02$ & & \\
\hline 胰腺 Pancreas & $0.52 \pm 0.11$ & $0.45 \pm 0.09$ & $0.52 \pm 0.13$ & $0.41 \pm 0.1$ & & \\
\hline 左肾上腺 Left adrenal & $0.0228 \pm 0.0059$ & $0.0198 \pm 0.0052$ & $0.0196 \pm 0.005$ & $0.0157 \pm 0.0038$ & $*$ & ** \\
\hline 右肾上腺 Right adrenal & $(0.0225)$ & $(0.0196)$ & $0.0211 \pm 0.0047$ & $0.0168 \pm 0.0035$ & & $*$ \\
\hline 左甲状腺 Left thyroid & $0.0056 \pm 0.0038$ & $0.0049 \pm 0.0034$ & $0.0089 \pm 0.0053$ & $0.0071 \pm 0.0044$ & $* *$ & $*$ \\
\hline 右甲状腺 Right thyroid & $0.0055 \pm 0.0038$ & $0.0048 \pm 0.0033$ & $0.009 \pm 0.0057$ & $0.0072 \pm 0.0046$ & $* *$ & $*$ \\
\hline 左卵巢 Left ovary & $0.0048 \pm 0.0024$ & $0.0041 \pm 0.0021$ & - & - & & \\
\hline 右卵巢 Right ovary & $0.0047 \pm 0.0024$ & $0.0041 \pm 0.0021$ & - & - & & \\
\hline 子宫 Uterus & $0.2 \pm 0.08$ & $0.17 \pm 0.06$ & - & - & & \\
\hline 左尿道球腺 Left bulbourethral gland & - & - & $(0.0282)$ & $(0.0215)$ & & \\
\hline 右尿道球腺 Right bulbourethral gland & - & - & $(0.0308)$ & $(0.0248)$ & & \\
\hline 阴茎 Penis & - & - & $0.29 \pm 0.1$ & $0.23 \pm 0.07$ & & \\
\hline 左睪丸 Left testis & - & - & $0.47 \pm 0.1$ & $0.37 \pm 0.07$ & & \\
\hline 右睪丸 Right testis & - & - & $0.46 \pm 0.09$ & $0.36 \pm 0.06$ & & \\
\hline 左精囊腺 Left seminal vesicle & - & - & $(0.09)$ & $(0.07)$ & & \\
\hline 右精囊腺 Right seminal vesicle & - & - & $0.09 \pm 0.03$ & $0.07 \pm 0.02$ & & \\
\hline 前列腺 Prostate & - & - & $0.07 \pm 0.02$ & $0.05 \pm 0.02$ & & \\
\hline
\end{tabular}

$P_{1}$ :脏器重量比较, $* P_{1}<0.05, * *: P_{1}<0.01$ 。 $P_{2}$ :脏器系数比较, $* P_{2}<0.05, * *: P_{2}<0.01$; “一”由于无对应值产生的缺省; 由于数据非正态分布用“(中位数)” 描述数据的集中趋势。

$P_{1}$ : Comparison of organ weight, ${ }^{*}: P_{1}<0.05,{ }^{* *}: P_{1}<0.01 ; P_{2}$ : Comparison of organ coefficient, $*: P_{2}<0.05, * *: P_{2}<0.01 ;$ Hyphens in the table are missing values because of no corresponding date; Describe the central tendency of the data using Median because data showed non-normal distribution. 
表 4 树鼠主要脏器指标的 Pearson 相关分析

Table 4 Pearson correlation of major organs in tree shrew

\begin{tabular}{|c|c|c|c|c|c|c|c|c|}
\hline $\begin{array}{c}\text { 指标 } / P \text { 值 } \\
\text { Index/P level }\end{array}$ & $\begin{array}{c}\text { 体重 } \\
\text { Weight }\end{array}$ & $\begin{array}{l}\text { 心脏 } \\
\text { Heart }\end{array}$ & $\begin{array}{l}\text { 肺脏 } \\
\text { Lung }\end{array}$ & $\begin{array}{l}\text { 肝脏 } \\
\text { Liver }\end{array}$ & $\begin{array}{c}\text { 脾脏 } \\
\text { Spleen }\end{array}$ & $\begin{array}{c}\text { 左肾 } \\
\text { Lift Kidney }\end{array}$ & $\begin{array}{c}\text { 右肾 } \\
\text { Right Kidney }\end{array}$ & $\begin{array}{c}\text { 脑 } \\
\text { Brain }\end{array}$ \\
\hline 体重 Body weight & 1 & $0.635^{* *}$ & $0.464 * *$ & $0.420 * *$ & 0.074 & $0.701 * *$ & $0.689 * *$ & $0.360 * *$ \\
\hline 心脏 Heart & $0.635^{* *}$ & 1 & $0.490 * *$ & $0.271^{*}$ & 0.037 & $0.529 * *$ & $0.518^{* *}$ & $0.302 *$ \\
\hline 肺脏 Lung & $0.464 * *$ & $0.490 * *$ & 1 & $0.356^{* *}$ & 0.024 & $0.369^{* *}$ & $0.414 * *$ & 0.158 \\
\hline 肝脏 Liver & $0.420 * *$ & $0.271 *$ & $0.356^{* *}$ & 1 & 0.075 & $0.530 * *$ & $0.499 * *$ & -0.036 \\
\hline 脾脏 Spleen & 0.074 & 0.037 & 0.024 & 0.075 & 1 & -0.038 & 0.018 & 0.132 \\
\hline 左肾 Lift Kidney & $0.701 * *$ & $0.529 * *$ & $0.369^{* *}$ & $0.530^{* *}$ & -0.038 & 1 & $0.903 * *$ & $0.296^{*}$ \\
\hline 右肾 Right Kidney & $0.689^{* *}$ & $0.518^{* *}$ & $0.414^{* *}$ & $0.499 * *$ & 0.018 & $0.903 * *$ & 1 & $0.292 *$ \\
\hline 脑 Brain & $0.360 * *$ & $0.302 *$ & 0.158 & -0.036 & 0.132 & $0.296^{*}$ & $0.292 *$ & 1 \\
\hline
\end{tabular}

$n=70 ; *: P<0.05, * *: P<0.01$.

近年来, 树鼠作为人类疾病动物模型有了新的 发展。由于种系、性别、年龄、体重及饲养条件 等因素影响，进一步完善实验室驯养树鼣的生物

\section{参考文献:}

Cao XM. 1989. The seasonal variation of spermatogenesis in Tupaia belangeri chinensis. Zoological Research, 10(1): 16-21. [曹符梅. 1989. 树 鼠(Tupaia belangeri chinensis)精子发生的季节性变化. 动物学研究, 10(1): 16-21.]

Cai JH, Zhu WL, Xie J, Jia T, Wang R, Lian X, Wang ZK. 2010. Variations on length and weight of the digestive tract in Tupaia belangeri. Chinese Journal of Zoology, 45(1): 140-144. [蔡金红, 朱万龙, 谢静, 贾婷, 王睿, 练硝, 王政昆. 2010. 中缅树鼠消化道长度和重量变化. 动物学杂志, 45(1): 140-144.]

Derting TL, Bogue BA. 1993. Responses of the gut to moderate energy demands in a small herbivore (Microtus pennsylvanicus). Journal of Mammalogy, 74(1): 59-68.

Dong L, Lü BL, Lai R. 2012. Molecular cloning of Tupaia belangeri chinensis neuropeptide $\mathrm{Y}$ and homology comparison with other analogues from primates. Zoological Research, 33(1): 75-78. [董丽, 吕龙, 赖仞. 2012. 树鼠神经肽 $\mathrm{Y}$ 的分子克隆及其灵长类类似物的同源性比较. 动物 学研究, 33(1): 75-78.]

Gross JE, Wang Z, Wunder B. 1985. Effects of food quality and energy needs: Changes in gut morphology and capacity of Microtus ochrogaster. Journal of Mammalogy, 66(6): 661-667.

He BL, Liu RW, Chen LL, Zheng H, Li B. 2008. The measurement of weight of main organs and the correlation research of organ weight $\&$ body weight in Tree Shrew. Laboratory Animal Science, 25(5): 17-19. [ 何保丽, 刘汝文, 陈丽玲, 郑红, 李波. 2008. 树鼠脏器系数的测定及脏器重量与 体重相关性的探讨. 实验动物科学, 25(5): 17-19.]

He ZM. 2006. Determination of Biological Characteristics of Laboratory Rodents Data Technical Regulations. Beijing: China Standards Press, 53-54. [贺争鸣. 2006. 啮齿类实验动物生物学特性数据测定技术规程. 北京: 中国标准出版社, 53-54.]

Jiao JL, Li B, Li JT, Zeng H, Chen LL, Shen PQ, Zheng H. 2011. Comparative analysis of nutritional status in wild and laboratory
学数据 (体尺、脏器重量、血液生理及血液生化等 基础指标)，对其实验动物化研究及应用具有重要 意义。

domesticated tree shrews. Laboratory Animal and Comparative Medicine, 31(4): 290-292. [角建林, 李波, 李进涛, 曾河, 陈丽玲, 沈培清, 郑红. 2011. 野生和实验室树舄营养状况的对比分析. 实验动物与比较医学, 31(4): 290-292.]

Jiao JL, Liu RW, Chen LL, Li B, He BL, Zheng H, Shen PQ. 2009. The development and use of tree shrew resource and its standards research-the strategic discussion for laboratory animal resource development in China. Chinese Journal of Comparative Medicine, 19(7): 73-78. [角建林, 刘汝文, 陈丽玲, 李波, 何保丽, 郑红, 沈培清. 2009. 树鼣资源的开发利用与标 准化研究-我国实验动物资源建设发展战略探讨. 中国比较医学杂志, 19(7): 73-78.]

Jiang QF, Kuang DX, Tong PF, Sun XM, Dai JJ. 2011. Scale breeding of tree shrews and the establishment of breeding population. Laboratory Animal Science, 28(6): 35-38. [江勤芳, 匡德宣, 全品芬, 孙晓梅, 代解杰 2011. 树舄规模化繁殖及繁殖群的建立. 实验动物科学, 28(6): 35-38.]

Korn H. 1992. Intestine lengths of southern African savanna rodents and insectivores: Intra-and inter-specific comparisons. Journal of Zoology, 228(5): 455-460.

Li XT, Zhu WL, Liu X, Liu CY, Wang ZK. 2011. Seasonal changes of energy metabolism in Tupaia belangeri. Acta Theriologica Sinica, 31(3): 291-298. [李晓婷, 朱万龙, 刘金金, 刘春燕, 王政昆. 2011. 中缅树鼠能量 代谢的季节变化. 兽类学报, 31(3): 291-298.]

Li YC, Hu JS, Li CM, Wang MK, Zhang SQ, Ouyang M, He Y, Xu GS. 1994. The annual periodic variations of reproductive system in Tupaia belangeri chinensis. Journal of Yunnan University: Natural Sciences, 16(1): 75-80. [李永材, 胡健生, 李春茂, 王敏康, 张士强, 欧阳明, 何云, 徐国 枢. 1994. 成年树鼠生殖系统的年周期变化. 云南大学学报: 自然科学 版, 16(1): 75-80.]

Nie W, Fu B, O'Brien PC, Wang J, Su W, Tanomtong A, Volobouev V, Ferguson-Smith MA, Yang F. 2008. Flying lemurs: the 'flying tree shrews'? Molecular cytogenetic evidence for a Scandentia-Dermoptera sister clade. BMC Biology, 6: 18 . 
Peng YZ, Ye ZZ, Zou RJ, Wang YX, Tian BP, Ma YY, Shi LM. 1991. Biology of Chinese Tree Shrews (Tupaia belangeri chinensis). Kunming: Yunnan Science and Technology Press. [彭燕章, 叶智章, 邹如金, 王应祥, 田保平, 马原野, 施立明. 1991. 树鼠生物学. 昆明: 云南科技出版社.] Sun JX, An J, Lian J. 2009. Analysis of effect factors of the weight and coefficients in laboratory Animal. Laboratory Animal Science, 26(1): 49-51. [孙建新, 安娟, 连军. 2009. 影响实验动物脏器重量及脏器系数因素分 析. 实验动物科学, 26(1): 49-51.]

Shen PQ, Zheng H, Liu RW, Chen LL, Li B, He BL, Li JT, Ben KL, Cao XM, Jiao JL. 2011. Progress and prospect in research on laboratory tree shrew in China. Zoological Research, 32(1): 109-114. [ 沈培清, 郑红, 刘 汝文, 陈丽玲, 李波, 何保丽, 李进涛, 贲昆龙, 曹䈗梅, 角建林. 2011.
中国树獡实验动物化研究进展和展望. 动物学研究, 32(1): 109-114.]

$\mathrm{Xu}$ L, Fan Y, Jiang XL, Yao YG. 2013. Molecular evidence on the phylogenetic position of tree shrew (Tupaia belangeri). Zoological Research, 34(2): 70-76. [许凌, 范宇, 蒋学龙, 姚永刚. 2013. 树鼠进化分 类地位的分子证据. 动物学研究, 34(2): 70-76.]

Ye YS, Han YY, Huan ZQ, Lu CX, Jiang QF, Sun XM, Dai JJ. 2011. Measurement and analysis of the weight and coefficients of major organs in wild adult tree shrews. Chinese Journal of Comparative Medicine, 21(4): 62-65. [叶尤松, 罕园园, 黄璋琼, 陆彩霞, 江勤芳, 孙晓梅, 代解杰. 2011. 野生成年树䳔主要脏器重量及脏器系数的测定分析. 中国比较医 学杂志, 21(4): 62-65.] 